\title{
Assessment of Malaria Vector Control Measures (ITNs \& IRS) Utilization and Factors Affecting it in Adama District, East Shoa Zone, Oromia Region Ethiopia, 2018
}

\author{
Dechasa Nesga ${ }^{1}$, Debalke Abate ${ }^{1}$, Girma Birhanu' ${ }^{1}$, Addamu Addissie ${ }^{2}$ \\ ${ }^{1}$ Ethiopian Field Epidemiology Training Program Resident, School of Public Health, Addis Ababa University, Addis Ababa, Ethiopia \\ ${ }^{2}$ Department of Preventive Medicine, School of Public Health, Addis Ababa University, Addis Ababa, Ethiopia
}

Email address:

dechasa_n@yahoo.com (D. Nesga)

\section{To cite this article:}

Dechasa Nesga, Debalke Abate, Girma Birhanu, Addamu Addissie. Assessment of Malaria Vector Control Measures (ITNs \& IRS) Utilization and Factors Affecting it in Adama District, East Shoa Zone, Oromia Region Ethiopia, 2018. Journal of Family Medicine and Health Care. Vol. 6, No. 2, 2020, pp. 46-51. doi: 10.11648/j.jfmhc.20200602.13

Received: May 29, 2019; Accepted: July 9, 2019; Published: May 28, 2020

\begin{abstract}
About $75 \%$ of landmass of Ethiopia is malaria-endemic, and $65 \%$ (58.5 million) of the population is estimated to be at risk of malaria infection. Malarious areas are targeted to receive key malaria control interventions (LLITNs and IRS) and we aimed to assess the utilization of ITN/IRS and factors affecting it. We conducted Community based Cross sectional study in Adama district from January 1-30, 2018 among 818 households using Multi stage sampling system followed by Simple random sampling method in selecting districts and camps. Bi-variate and multivariate logistic regression was used to calculate the association. Of 818 visited HHs $73 \%$ were utilizing ITN regularly and sprayed by IRS in the past one year. The prevalence rate of malaria was found to be six percent. Having good awareness about malaria transmission and control measures (AOR 1.4 (1.1-2), sharing one bed with a member of 3-5 compared to not sharing (AOR=0.7 (0.4-0.9), family head educational level of higher level compared to illiterate ( $\mathrm{AOR}=4.8,95 \% \mathrm{CI} 1.1-6.6$ ), Employed family head Compared to Farmers (AOR=8.1, 95\% CI 7.8-10.7) are significantly associated with ITN utilization. ITN utilization was influenced by Lack of knowledge, lack of education and family sharing one bed. Increasing the knowledge of community on malaria transmission and prevention methods including ITN proper usage is important.
\end{abstract}

Keywords: Malaria, Control Method, Barrier of Utilization of ITN/IRS, Adama, 2018

\section{Introduction}

Malaria is a tropical disease with high morbidity and mortality resulting in high economic and social impacts. Even though it has no vaccination, it is an absolutely preventable and treatable vector-borne illness which is caused by protozoal parasite of the genus Plasmodium. [1]

According to World Health Organization report in 2016, an estimated 216 million cases of malaria occurred worldwide in which $90 \%$ was from the WHO African Region. Of the 91 countries that had an indigenous malaria case in 2016, a decrease in malaria cases of more than $20 \%$ compared with 2015 was estimated in 16 countries, while an increase of a similar magnitude was estimated in 25 countries. The WHO regions of the Americas and Africa accounted for nearly $70 \%$ of the countries that had increases of more than $20 \%$ in 2016 compared with 2015. [2]

World Health Organization (WHO) has formulated different strategies to combat the high prevalence of Malaria in the world. The most commonly used methods to prevent mosquito bites are sleeping under an Insecticide Treated Nets and spraying the inside walls of a dwelling with an insecticide - an intervention known as IRS. Use of ITNs has been shown to reduce malaria case incidence rates by $50 \%$ in a range of settings, and to reduce malaria mortality rates by $55 \%$ in children aged less than 5 years in sub-Saharan Africa. Between 2014 and 2016, a total of 582 million insecticidetreated mosquito nets (ITNs) were reported as having been delivered globally, of which almost 505 million ITNs (87\%) were delivered to countries in sub-Saharan Africa. [2]

In Ethiopia malaria constitutes a major public health problem and impediment to socio-economic development 
where about $75 \%$ of landmass of the country is malariaendemic and $65 \%$ (58.5million) of the population is estimated to be at risk of malaria infection.[3] According to the national strategy for malaria control, areas lower than 2,000 meters in altitude were considered to be malariaendemic area and targeted to receive key malaria control interventions (long-lasting insecticidal nets (LLINs), \& indoor residual spraying (IRS)) stating a national goal of $100 \%$ household LLINs coverage with a mean of two LLINs per household. [4]

In 2015, Ethiopian National malaria indicators survey indicated LLTINs utilization in Oromia region is found to be $59 \%$ which is less than expected. [5] As different study showed the utilization of ITN may be affected by different personal, social or environmental barriers. Under-five children and pregnant women are high risk groups and priority should be given. The study done in Mihrab Abaya Gamo Gofa zone shows ITN utilization among less than five children was about 37.2\%. [6] Education level of the House Hold, awareness about ITNs, number of sleeping rooms in the $\mathrm{HH}$, individual ITN-color preference, presence of children aged under 5 years in the HH, IRS status of the $\mathrm{HH}$, awareness of malaria prevention, family size of the $\mathrm{HH}$, and occupational status of the $\mathrm{HH}$ head are reported as affecting factors in different previous study. [7 8] The study supported by President's malaria initiative and conducted in five districts of Jimma Zone in 2015 shows, the shape of ITN, low risk perception due to seasonality of malaria, saving nets for future use, decreased awareness, negligence and perceived low efficacy of LLITN are the major barriers. Again, the study conducted in Gambella also shows, sleeping in a member of more than three is more likely to use bed nets. [8] Other study done in Areka town Southern Ethiopia in 2015 reports among Households those are using ITN properly, $50.4 \%$ of them have good awareness about ITN utilization and similarly the study in west Gojjam zone done in 2014 shows knowledge has a significant association with ITN utilization. [10, 11] Adama District is one of Oromia region zone with many malaria risk factors and as information received from the district malaria expert report shows, in different time the distribution of ITNs and IRS was done. Despite this, Malaria illness remains the leading cause of morbidity indicating either low ITN/IRS coverage or poor ITN utilization in this district. Thus, this study helps to discloses the level of ITN utilization in Adama district and affecting factors thereby providing credible information for stake holder to achieve national malaria control strategic plan.

\section{Methods \& Materials}

\subsection{Study Area \& Period}

This study was conducted in Adama District from January $1-15,2018$. It is one of the 12 woredas in East Shoa Zone of
Oromia regional State, around $100 \mathrm{~km}$ East of Addis Ababa having 41 rural Kebeles, 4 Suburban kebeles (the lowest division of administrative area) and 15 Factory camps with estimated total population of 193,885. Geographically Adama district found in Rift Valley and 95\% of the villages in the District are located in the low land while $90 \%$ of populations are at risk of malaria transmission. Morbidity report from health facilities showed that, malaria is among the ten top causes of morbidity diseases.

\subsubsection{Study Design}

Community based Cross-sectional study design was employed.

\subsubsection{Source Population}

The source population was all households in Adama district including 15 Factory camps.

\subsubsection{Sampling Units}

Our study unit was systematically selected Households owned at least one any type of ITNs and /or households sprayed by indoor insecticide chemicals at least one times in the year in the selected Kebeles and camps.

\subsection{Sample Size Calculation}

\subsubsection{Sampling Technique}

The study units were identified by multi stage sampling technique followed by Simple random sampling method to identify primary sample and systematic sampling the final study unit. List of all Kebeles and camps in the district was used as sampling frame. The source of population was clustered as Malarias Kebele (35) and Camps (15) with a $\mathrm{HH}$ population of $84 \%$ and $16 \%$ respectively. All malarias Kebeles and camps identified by the district health office were selected and taken as primary sampling unit. Twenty percent of malarias Kebeles and $20 \%$ of factory camps were selected by Lottery method using the list of each cluster as sampling frame. Hence, seven malarious Kebeles and Three Factory camps were selected. On the second stage, households in each randomly selected Kebeles and camps were used as secondary sampling units. The number of households which to be selected from each identified Kebeles \& Camps were determined by the households (population) proportion of respective Kebeles. Furthermore, the first house hold was selected by simple random sampling and the others were selected systematically by counting every nine houses. In cases when the selected Household is not fulfilled inclusion criteria of the study the next house was selected.

\subsubsection{Data Collection Procedure}

We used structured questionnaire which were adopted from different literatures and customized. It was translated to Local Language from English Version for interviewers' administered house to houses data collection. Family head or a family member who are constantly living in the house and above 18 ysr old was selected for face to face interview. 


\section{Result}

\subsection{Socio Demographic Information}

In our study, we achieved $100 \%$ respondent rate and since our study was fully focused on the households with at least one ITN or/ sprayed with indoor Insecticide, $100 \%$ of them owned at least one ITN (any type) and 596 (73\%) of the visited house were sprayed with Indoor insecticide residual in the past one year. The median age of the participant was 33 yrs (IQR=17). Among the total 818 visited households with family member of 3,537 about 209 people was treated for Malaria illness in the last year (2017) which accounts the prevalence of $6 \%$. Of 818 participants 516 (63\%) were Female population and the educational level of most of the participant, 341 (42\%), was Elementary level. In the study area the major source of family income was Farming which accounts about $565(69 \%)$.

In the study area $578(71 \%)$ of the House Holds (HHs) has habits of sharing one beds with family members among which more than half of them 315 (54\%) HHs family Members sleeps by sharing a single bed or sleeping area with three to five family members of the same HH. Of 818 visited HHs under five children founds in 387 (47\%) and 342 (88\%) of them sleeps with their parents on the same bed. Among visited houses $583(71 \%)$ HHs were made of corrugated-iron houses, while the majority $782(96 \%)$ had separate sleeping room and 497 (61\%) of them had two and more sleeping area (Table 1).

Table 1. Sleeping habits and housing conditions of study participants in Adama district, East Shoa zone Oromia region, Ethiopia, 2018.

\begin{tabular}{|c|c|c|c|c|c|}
\hline Variables & Category & Frequency & Percent & $95 \%$ LCI & $95 \%$ UCI \\
\hline \multirow{2}{*}{ Family shares one bed $(n=818)$} & Yes & 578 & $71 \%$ & $67 \%$ & $74 \%$ \\
\hline & No & 240 & $29 \%$ & $26 \%$ & $33 \%$ \\
\hline \multirow{3}{*}{$\begin{array}{l}\text { Number of family member shares one } \\
\text { bed }(n=578)\end{array}$} & Two and less & 259 & $45 \%$ & $34 \%$ & $48 \%$ \\
\hline & 3-5 people & 315 & $54 \%$ & $41 \%$ & $57 \%$ \\
\hline & More than five & 4 & $1 \%$ & $0.5 \%$ & $1.5 \%$ \\
\hline \multirow{2}{*}{ Housing Conditions $(\mathrm{n}=818)$} & Thatch/leaf & 235 & $29 \%$ & $26 \%$ & $32 \%$ \\
\hline & Corrugated Iron & 583 & $71 \%$ & $68 \%$ & $74 \%$ \\
\hline Under five children present $(\mathrm{n}=818)$ & No & 431 & $53 \%$ & $49 \%$ & $56 \%$ \\
\hline \multirow{3}{*}{$\begin{array}{l}\text { Sleeping habits of under five children } \\
(n=387)\end{array}$} & All children under 5years Sleep together & 42 & $11 \%$ & $7 \%$ & $13 \%$ \\
\hline & Sleep with both parents & 342 & $88 \%$ & $83 \%$ & $91 \%$ \\
\hline & Sleep with other family member & 3 & $1 \%$ & $0.8 \%$ & $1.6 \%$ \\
\hline \multirow{2}{*}{ Separate Bed rooms $(n=818)$} & Yes & 782 & $96 \%$ & $94 \%$ & $97 \%$ \\
\hline & No & 36 & $4 \%$ & $3 \%$ & $6 \%$ \\
\hline \multirow{2}{*}{$\begin{array}{l}\text { Number of Sleeping Rooms/Bed } \\
(\mathrm{n}=818)\end{array}$} & One & 321 & $39 \%$ & $36 \%$ & $43 \%$ \\
\hline & Three and more & 127 & $16 \%$ & $13 \%$ & $18 \%$ \\
\hline
\end{tabular}

\subsection{Owner Ship \& Utilization of ITNs}

Of 818 participants, more than half of them $519(63 \%)$ have more than one ITN (any type). House to house inspection was conducted during our assessment to observe the proper use of existing ITN for family. This was measured by whether the bed nets are hanged over the bed properly or not. Among 595 (73\%) households found to have at least one bed with bed net, about 178 (30\%) of them was observed that not properly hanged as it prevents insect bite. Of the total HHs observed, only 417 (51\%) of the households hanged ITN properly on the sleeping area (Figure 1).

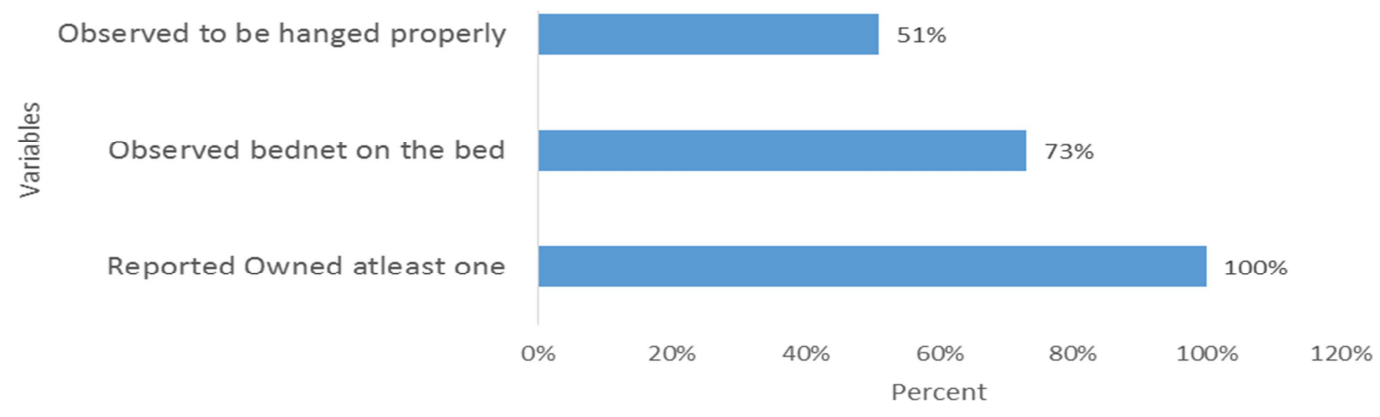

Figure 1. Comparison of Reported and Observed Bed Net owner ship Vs utilization.

The most of bed nets found in house hold was LLINs type $542(66 \%)$ and among those who owned retreat able types, $276(36 \%)$, only $3(1 \%)$ of them were retreated at most one per year. Lack of information and chemical was the main reason that the community complain for why they not retreat their bed nets regularly. In addition to using bed nets for malaria vector control in home, $596(73 \%)$ of the visited households were sprayed with IRS in the past one year.

Even though most of the visited family has more than two ITN (any type), when we compare the ITN owner ship and utilization, among those owns more than one ITN, 595 (63\%), about $159(27 \%)$ were utilizing only one ITN and among 299 households owned only one ITN, 83 (28\%) of them were not using the existing ITN (Table 2). 
Table 2. ITN Owner Ship and Utilization in Adama District, East Shoa, Oromia, 2018

\begin{tabular}{|c|c|c|c|c|c|}
\hline Variables & Category & Frequency & Percent & Wilson 95\% LCL & Wilson $95 \%$ UCL \\
\hline \multirow{4}{*}{ Number of ITN in HH $(n=818)$} & One & 299 & $37 \%$ & $33 \%$ & $40 \%$ \\
\hline & Two & 386 & $47 \%$ & $44 \%$ & $51 \%$ \\
\hline & Three and above & 133 & $16 \%$ & $14 \%$ & $19 \%$ \\
\hline & One & 377 & $46 \%$ & $43 \%$ & $50 \%$ \\
\hline \multirow{3}{*}{$\begin{array}{l}\text { Number of sleeping Beds observed with } \\
\text { Bed net }(n=818)\end{array}$} & Two & 199 & $24 \%$ & $22 \%$ & $27 \%$ \\
\hline & Three and above & 19 & $2 \%$ & $1 \%$ & $4 \%$ \\
\hline & No sleeping places with bed net & 223 & $27 \%$ & $24 \%$ & $30 \%$ \\
\hline \multirow{2}{*}{ Types of ITN $(n=818)$} & Re treatable & 276 & $34 \%$ & $31 \%$ & $37 \%$ \\
\hline & LLITNs & 542 & $66 \%$ & $63 \%$ & $69 \%$ \\
\hline \multirow{2}{*}{ Retreated regularly $(\mathrm{n}=276)$} & Yes & 3 & $1 \%$ & $0 \%$ & $3 \%$ \\
\hline & No & 273 & $99 \%$ & $97 \%$ & $100 \%$ \\
\hline \multirow{2}{*}{ ITN hanged properly $(n=595)$} & Yes & 417 & $70 \%$ & $66 \%$ & $74 \%$ \\
\hline & No & 178 & $30 \%$ & $26 \%$ & $34 \%$ \\
\hline \multirow{2}{*}{ Shapes of bed nets $(\mathrm{n}=818)$} & Conical & 9 & $1 \%$ & $1 \%$ & $2 \%$ \\
\hline & Rectangular & 809 & $99 \%$ & $98 \%$ & $99 \%$ \\
\hline \multirow{2}{*}{ IRS sprayed with in one year $(\mathrm{n}=818)$} & Yes & 596 & $73 \%$ & $70 \%$ & $76 \%$ \\
\hline & No & 222 & $27 \%$ & $24 \%$ & $30 \%$ \\
\hline \multirow{4}{*}{$\begin{array}{l}\text { Under five children in house member } \\
(\mathrm{n}=818) \\
<5 \text { Children sleep under Bed net last night } \\
(\mathrm{n}=387)\end{array}$} & Yes & 387 & $47 \%$ & $44 \%$ & $51 \%$ \\
\hline & No & 431 & $53 \%$ & $49 \%$ & $56 \%$ \\
\hline & Yes & 221 & $57 \%$ & $52 \%$ & $62 \%$ \\
\hline & No & 166 & $43 \%$ & $38 \%$ & $48 \%$ \\
\hline \multirow{2}{*}{$\begin{array}{l}\text { Pregnant women in House Member } \\
(\mathrm{n}=818)\end{array}$} & Yes & 110 & $13 \%$ & $11 \%$ & $16 \%$ \\
\hline & No & 708 & $87 \%$ & $84 \%$ & $89 \%$ \\
\hline \multirow{2}{*}{ PLW used ITN last night (n=110) } & Yes & 67 & $61 \%$ & $51 \%$ & $70 \%$ \\
\hline & No & 43 & $39 \%$ & $30 \%$ & $49 \%$ \\
\hline
\end{tabular}

\subsection{Factors that Influencing ITN Utilization}

Among factors those are suspected to have positive or negative impacts on ITN utilization and cross tabulated with households utilizing at least one bed nets regularly, the variables those have a $p$ value of 0.2 and less were entered into multivariate logistic regression to avoid possible confounders. In multivariate analysis the variables with a $\mathrm{p}$ value of less than 0.05 are considered to have significant association with ITN utilization. Accordingly, Multivariate analysis showed that the participants who have good awareness about malaria transmission and control measures utilizes ITN properly 1.4 times more than the counterpart
(95\% CI, 1.1, 2; P value 0.0123). The family who have a habit of sharing one bed with a member of 3-5 were $30 \%$ less likely to utilize ITN properly than those sleep alone $(95 \% \mathrm{CI}$ $0.7(0.4,0.9)$; $\mathrm{p}$ value 0.0396$)$. Households with family head educational level of higher level were found to utilize ITN properly 4.4 times $(\mathrm{AOR}=4.4,95 \%$ CI $1.1-20.4$; $\mathrm{P}$ value 0.0473 ) than illiterate family head and $\mathrm{HH}$ with Employed family head utilizes ITN 8.4 times (AOR $=8.4,95 \%$ CI 2.9 24.8; $\mathrm{P}$ value 0.0001$)$. In addition, the households where there were under five children were observed 1.9 times more likely to use ITN than those with had no under-five age children (Table 3).

Table 3. Multi variate analysis of ITN utilization influencing factors, Adama District, 2018.

\begin{tabular}{|c|c|c|c|c|c|c|c|}
\hline \multirow{2}{*}{$\mathbf{S} / \mathbf{n}$} & \multirow{2}{*}{ Variables } & \multirow{2}{*}{ Category } & \multicolumn{5}{|c|}{ Utilize at least one bed net last night } \\
\hline & & & Yes $(n=595)$ & No $(n=223)$ & COR $(95 \%$ CI) & AOR $(95 \%$ CI) & P Value \\
\hline & \multirow{3}{*}{$\begin{array}{l}\text { Knowledge level } \\
(\mathrm{n}=818)\end{array}$} & Good & $473(80 \%)$ & $163(73 \%)$ & $2.6(1.8,3.6)$ & $1.5(1.1,2.21)$ & $0.0468 *$ \\
\hline & & Poor & $122(20 \%)$ & $60(27 \%)$ & Reference & 1 & \\
\hline & & Two and less & $207(35 \%)$ & $52(22 \%)$ & $0.96(0.6,1.5)$ & $1.3(0.8,2.3)$ & 0.2647 \\
\hline & \multirow{3}{*}{$\begin{array}{l}\# \text { of Person share } \\
\text { one bed }(n=818)\end{array}$} & 3-5 people & $192(32 \%)$ & $123(55 \%)$ & $0.4(0.3,0.6)$ & $0.7(0.4,0.9)$ & $0.0396 *$ \\
\hline & & More than 5 & $3(1 \%)$ & $1(1 \%)$ & $0.7(0.1,7.1)$ & $1.1(0.1,11.9)$ & 0.9488 \\
\hline & & No sharing bed & $193(32 \%)$ & $47(21 \%)$ & Reference & 1 & \\
\hline & \multirow{4}{*}{$\begin{array}{l}\text { Education } \\
(\mathrm{n}=818)\end{array}$} & Higher level & $42(7 \%)$ & $2(1 \%)$ & $16.6(3.9,70)$ & $4.4(1.1,20.4)$ & $0.0473 *$ \\
\hline & & Secondary & $152(26 \%)$ & $5(2 \%)$ & $24(9.5,60.5)$ & $10.4(3.9,27.7)$ & $0.0000 *$ \\
\hline & & Primary & $247(41 \%)$ & $94(42 \%)$ & $2(1.5,2.9)$ & $1.4(1.2,2.1)$ & $0.0055^{*}$ \\
\hline & & Illiterate & $154(26 \%)$ & $122(55 \%)$ & Reference & 1 & \\
\hline & \multirow{5}{*}{$\begin{array}{l}\text { Occupation } \\
(\mathrm{n}=818)\end{array}$} & Employed & $128(22 \%)$ & $4(1.5 \%)$ & $19.4(17,23)$ & $8.4(2.9,24.8)$ & $0.0001 *$ \\
\hline & & Daily Laborer & $65(11 \%)$ & $5(2 \%)$ & $7.8(3.1,10.8)$ & $4.3(1.7,11.3)$ & $0.0028 *$ \\
\hline & & Trading & $44(7 \%)$ & $1(0.5 \%)$ & $26.6(23.1,34.4)$ & $11.1(1.5,84.2)$ & $0.0203 *$ \\
\hline & & House wife & $6(1 \%)$ & $1(0.5 \%)$ & $3.6(0.4,30.2)$ & $2.6(0.3,23.2)$ & 0.3981 \\
\hline & & Farmer & $352(59 \%)$ & $211(95 \%)$ & Reference & 1 & \\
\hline & \multirow{2}{*}{$\begin{array}{l}<5 \text { children in the } \\
\mathrm{HH}(\mathrm{n}=818)\end{array}$} & Yes & $291(49 \%)$ & $96(43 \%)$ & $1.3(0.9,1.7)$ & $1.9(1.2,2.8)$ & $0.0025 *$ \\
\hline & & No & $304(51 \%)$ & $127(57 \%)$ & Reference & 1 & \\
\hline
\end{tabular}

Note: * Variables those are significantly associated with ITN utilization. 


\section{Discussion}

The national strategy for malaria control recommended $100 \%$ of ITN coverage with high proportion and mean of two LLTINs per households in malarious area [4]. Parallel to this recommendation, most of the dwellers of Adama district $(66 \%)$ were found to own LLITNs. The cross tabulation of IRS with types of ITN in the household showed that families those owned LLITNs were $31 \%$ less likely to use IRS for dwelling house wall spray $(\mathrm{COR}=0.6995 \%$ CI $0.5-0.9, \mathrm{P}$ value 0.0318 ). However, only $63 \%$ of households owned two and more ITN (any types) which is too much lower than national recommendation. This finding is higher than the study findings done in Harari regional state in 2015 [12] which were $57.9 \%$ and are lower than the findings in Gambella Region which is $68.4 \%$ in 2014 [13]. As we observed during data collection, the ITN found in the most of HHs were very aged which was distributed three and four years back. Because of handling problem and ageing of the nets, most of observed ITNs were damaged. Hence, they throw it away after it torn without replacement. Probably this is the reason why most of households were observed having less than the mean recommended number of ITN per house.

Despite $100 \%$ owning of any types of ITN or spraying IRS in the district, we identified the overall Malaria prevalence among visited households to be $6 \%$ which is lower than prevalence reported in 2013 study in this district (13.17\%). [11] This shows $50 \%$ declining of malaria infection in the district within four years which a line with the national PMI target. On the other hand, the existence of this much prevalence of malaria, despite high coverage and better utilization of ITN reported, may be due to ITN proper utilization (hanging) problem observed in the district which was out of visited households only $51 \%$ was hanged properly. In addition, it may be due to aged or worn out of existing ITN which lacks its integrity and effectiveness to prevent Mosquito bite.

The overall utilization in Adama district in 2017 was about $73 \%$ which is higher than the overall utilization in Oromia region in 2015 [14] and similar with the utilization level in Harari region in 2014 [12]. ITN utilization among under five children (57\%) and pregnant women 61\%) in Adama district was higher than reports from national malaria indicator survey in 2015 which was $43.3 \%$ and 41.7 respectively in Oromia region [14]. ITN utilization among under five children in Adama district is also higher than the study results in Mihrab Abaya, Gamo Gofa zone in 2016 which was only $37.2 \%$ [15]. This difference may be due increased community awareness created by health extension workers about ITN utilization and prioritization. But, still this finding is not satisfactory regarding Children and pregnant mother prioritization which needs improvement because this is very vulnerable age groups among the community.

On the other hand, significant association was observed among $\mathrm{HH}$ those have at least one under five children and ITN utilization in our study area in which Families where there were under five children utilize 1.9 times $(\mathrm{AOR}=1.9,95 \% \mathrm{CI}$ 1.2- $2.8, \mathrm{p}$ value 0.0025 ) more likely to utilize ITN than the counterpart. This may also the result of improved community awareness on vulnerability of under five children for malaria infection and so that they tend to use ITN for their children.

The odds of utilizing ITN was 1.5 times higher among participants those have good awareness about Malaria transmission and control measures compared to those who have poor awareness. This finding is supported by the similar study done in West Gojjam zone in 2014 (AOR=3, 95\% $\mathrm{CI}=1.7-5.4$ ) [6], In Mihrab Abaya Gamo Gofa Zone in 2016 $(\mathrm{AOR}=2.51,95 \%$ CI 1.17, 5.37) [10] and in Itang, Gambella zone which shows $72 \%$ less likely to use ITN among those have poor knowledge $(\mathrm{AOR}=0.28,95 \% \mathrm{CI}$ 0.15-0.53) [13]. Families where 3-5-member shares one bed were found to be $30 \%$ less likely to use ITN compared to those not shares one bed. This aligns with the study done in Itang, Gambella zone in $2016(\mathrm{AOR}=0.19,95 \% \mathrm{CI} 0.1-0.38)$ [13]. On the other hand, employed Family head is 8.4 times more likely to use ITN properly than Farmers (AOR $=8.4$, 95\% CI 2.9-24.8, p value 0.0001$)$. This may be due to those Employed peoples are more educated than the farmers and have better awareness about malaria transmission and control measures.

\section{Conclusion}

In our study area despite high coverage and utilization of ITN/IRS, the prevalence of Malaria was very high. Most of the households owned the minimum requirement of ITN as national target. Improper ITN utilization was observed which was significantly associated with Knowledge level of family head, Occupation of family head, number of family members sharing one bed, and Educational level of family head. On the other hand our study reveals that ITN utilization was not influenced by number of ITN, Family size and monthly income of the family in Adama District.

\section{Recommendation}

To improve ITN utilization and decrease malaria transmission in the district, continuous health education including demonstration of how to hang nets is important. In addition increasing the knowledge level of the community on malaria transmission and control measures so that they know the importance of ITN is critical activities for malaria control. Again Continuous house to house observation of proper bed net utilization could increase the utilization of ITN/IRS.

\section{References}

[1] CDC 24/7: saving lives, protecting people, July 20, 2017.

[2] WHO, World malaria report, 2017 ISBN 978-92-4-156552-3.

[3] Ethiopian Health and Nutrition Research Institute (EHNRI): Public Health Emergency Management Guideline for Ethiopia, 2012. 
[4] President's malaria initiative Ethiopia malaria operational plan FY 2017.

[5] WHO, World Malaria Report, 13 December 2016.

[6] Dawit Siraw, Assessment of ownership and factors affecting utilization of Insecticide Treated bed nets in Dembecha woreda, West Gojjam zone, Ethiopia from September 2013 to June 2014.

[7] Tadele G, Samuel A, Adeba E. Replacement of long lasting insecticide treated nets in malarious kebeles of Gida Ayana district, East Wollega zone, Ethiopia, 2014; 3: 162-166.

[8] Gobena T, Berhane Y, Worku A. Low long-lasting insecticide nets (ITNS) use among.

[9] Deresse Legessa et al. Knowledge, Attitude and Practice towards Malaria and Associated Factors in Areka Town, Southern Ethiopia, 2015.

[10] Admasu Tasew et. Al. Factors influencing the ownership and utilization of long-lasting insecticidal nets for malaria prevention in Ethiopia, 2014.

[11] Gezehagne Sebsibe, 2013: Current status of the prevalence of malaria and its associations with knowledge, awareness, anthropogenic factors, climate and abo blood groups in Adama district, Oromia regional state, Ethiopia.

[12] Zelalem T/Mariam, et. al, Ownership and utilization of insecticide-treated nets (ITNs) for malaria control in Harari National Regional State, Eastern Ethiopia, 2015.

[13] Aklilu Habte, 2016 Insecticide-treated net ownership and utilization and factors that influence their use in Itang, Gambella region, Ethiopia: cross-sectional study.

[14] Ethiopian National malaria indicators survey, 2015.

[15] Amha Admassie, et. al: ITN utilization and associated factors among under five children in mihrab Abaya, Gamo Gofa Zone Ethiopia, 2016. 